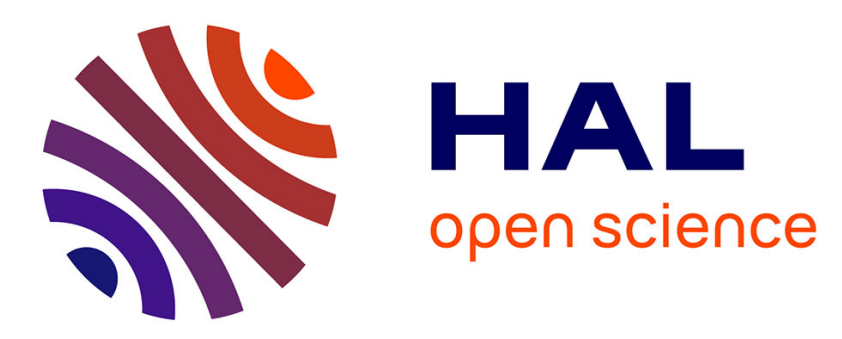

\title{
Microvilli-derived Extracellular Vesicles Govern Morphogenesis in Drosophila wing epithelium
}

Ilse Hurbain, Anne-Sophie Macé, Maryse Romao, Lucie Sengmanivong, Laurent Ruel, Renata Basto, Pascal P Thérond, Graça Raposo, Gisela D'angelo

\section{To cite this version:}

Ilse Hurbain, Anne-Sophie Macé, Maryse Romao, Lucie Sengmanivong, Laurent Ruel, et al.. Microvilli-derived Extracellular Vesicles Govern Morphogenesis in Drosophila wing epithelium. 2021. hal-03414813

\section{HAL Id: hal-03414813 https://hal.science/hal-03414813}

Preprint submitted on 4 Nov 2021

HAL is a multi-disciplinary open access archive for the deposit and dissemination of scientific research documents, whether they are published or not. The documents may come from teaching and research institutions in France or abroad, or from public or private research centers.
L'archive ouverte pluridisciplinaire HAL, est destinée au dépôt et à la diffusion de documents scientifiques de niveau recherche, publiés ou non, émanant des établissements d'enseignement et de recherche français ou étrangers, des laboratoires publics ou privés. 


\section{Microvilli-derived Extracellular Vesicles Govern Morphogenesis in}

\section{Drosophila wing epithelium}

3 Ilse Hurbain ${ }^{1,2}$, Anne-Sophie Macé ${ }^{1,2}$, Maryse Romao ${ }^{1,2}$, Lucie Sengmanivong ${ }^{1,2}$, Laurent

4 Ruel $^{3}$, Renata Basto ${ }^{1}$, Pascal P.Thérond ${ }^{3}$, Graça Raposo ${ }^{1,2}$ and Gisela D’Angelo ${ }^{1,2, *}$.

$6 \quad{ }^{1}$ Institut Curie, PSL Research University, CNRS, UMR144, 26 rue d'Ulm, 75248 Paris Cedex 7 05, France.

$8 \quad 2$ Institut Curie, PSL Research University, CNRS, UMR144, Cell and Tissue Imaging Facility 9 (PICT-IBiSA), 26, rue d'Ulm, 75248 Paris Cedex 05, France.

103 Université Côte d'Azur; UMR7277 CNRS; Inserm U1091; Institute of Biology Valrose 11 (iBV); Parc Valrose ; 06108 Nice cedex2, France. gisela.dangelo@curie.fr

\section{ABSTRACT}

The regulation and coordination of developmental processes involves the secretion of morphogens and membrane carriers, including extracellular vesicles, which facilitate their transport over long distance. The long-range activity of the Hedgehog morphogen is conveyed by extracellular vesicles. However, the site and the molecular basis of their biogenesis remains unknown. By combining fluorescence and electron microscopy combined with genetics and cell biology approaches, we investigated the origin and the cellular mechanisms underlying extracellular vesicle biogenesis, and their contribution to Drosophila wing disc development, exploiting Hedgehog as a long-range morphogen. We show that microvilli of Drosophila wing disc epithelium are the site of generation of small extracellular vesicles that transport Hedgehog across the tissue. This process requires the Prominin-like protein, whose activity, together with 
interacting cytoskeleton components and lipids, is critical for maintaining microvilli integrity and function in secretion. Our results provide the first evidence that microvilli-derived extracellular vesicles contribute to Hedgehog long-range signaling activity highlighting their physiological significance in tissue development in vivo.

Hedgehog $(\mathrm{Hh})$ is a well-known long-range morphogen controlling tissue patterning and cell differentiation during embryonic development ${ }^{1}$. In the Drosophila wing imaginal disc epithelium, Hedgehog produced in posterior cells, signals across the anterior (A)/posterior (P) boundary to induce the expression of short- [i.e. engrailed (En), Patched (ptc)], and long-range [i.e. decapentaplegic $(d p p)$ ] target genes in anterior recipient cells. Different modes of carriermediated Hh long-range signaling have been proposed ${ }^{1}$, including extracellular vesicles (EVs), that arise either from fusion of multivesicular body (MVB) with the plasma membrane (exosomes), or from budding of the plasma membrane (ectosomes also termed microvesicles ${ }^{2}$. Evidences have been provided to suggest that both types of EVs, whose nature is tightly dependent on the site of Hh secretion (apical ${ }^{3-5}$ and/or basolateral secretion ${ }^{6}$ ) could mediate $\mathrm{Hh}$ long-range activity in Drosophila epithelia ${ }^{4,7-9}$. The apical membrane of epithelial cells is characterized by the presence of microvilli that have been shown to give rise to EVs ${ }^{10,11}$. However, our understanding of the mechanisms of microvilli-EV biogenesis, and their potential contribution to intercellular communication during development is presently unclear. This is mainly due to the fact that current models describing the origin of microvilli-derived EVs, focus on the relationship between the occurrence of microvilli-derived EVs and specific developmental stages ${ }^{10}$. These studies, which are based on ultrastructural, biochemical and pharmacological observations, reveal only part of the mechanisms of EV biogenesis and do not directly investigate the functional consequences during the developmental process in vivo. To understand the relationship between microvilli, EV biogenesis and their potential significance 
in development, we investigated the origin of EVs, the molecular mechanisms underlying their biogenesis, and their function in Hh long-range signaling using Drosophila wing imaginal disc epithelium as a paradigm.

Microvilli that are globally aligned at the apical membrane of epithelial cells, bear proteins of the Prominin family ${ }^{12-14}$, and have been shown, in mammalian cells, to give rise to Prominin-containing EVs ${ }^{10,11}$. Considering this, we examined whether the sorting of $\mathrm{Hh}$ to microvilli and their ability to generate EVs could be prerequisites for the EV-mediated deployment of Hh long-range signaling in the Drosophila wing imaginal disc (Fig. 1a). To investigate the formation of microvilli-derived EVs, we monitored the Prominin-like (PromL) protein that has been reported, upon overexpression, to distribute to apical protrusions of Drosophila wing imaginal discs ${ }^{15}$. To validate the newly generated antibodies raised against the PromL protein (methods and Supplementary Fig. 1a), we analyzed their subcellular distribution in the dorsal wing disc compartment expressing GFP-tagged PromL ${ }^{15}$ driven by apterous-Gal4 driver (ap>PromL-GFP) and compared to the ventral wild-type compartment within the same discs. Endogenous PromL signals massively colocalized with PromL-GFP, distributed at the uppermost apical surface above aPKC, E-cadherin (E-cad) and Dlg (apical, subapical and basolateral markers respectively), was not detected at the basolateral where PromL-GFP was previously observed ${ }^{15}$, and did not overlap with GFP-tagged Viking (GFPVkg, a collagen IV type molecule that labels the basement membrane) ${ }^{16}$ (Fig. 1b; Supplementary Fig 1b,c). In addition, PromL fully colocalized with Cad99c, a specific marker of microvilli of ovarian follicle and wing imaginal disc epithelial cells ${ }^{17,18}$ (Supplementary Fig. 1d). Overall, these results show that PromL, is associated with the apical compartment of epithelial wing disc cells, consistent with an association and distribution to microvilli. 
Next, we functionally tested the impact of PromL protein on microvilli architecture and integrity through knock down (KD) using RNA interference (RNAi) driven by ap> or hedgehog-Gal4 (hh>) to target dorsal or posterior cells respectively. We found that in both genetic backgrounds PromL signal was specifically abrogated (Fig. 1b; Supplementary Fig. 2a). However, cell polarity (as revealed by aPKC, E-cad and Dlg staining) was not perturbed and apoptosis levels were not increased (as revealed by caspase III staining), indicating an absence of tissue stress in such condition (Fig. 1b; Supplementary Fig. 2b,c). In addition, the wt compartment, displayed the usual dot-like pattern of PromL reflecting a correct organization of microvilli at the apical plasma membrane (Fig. 1a,b; Supplementary Fig. 1e). We conclude that PromL specifically localised and confined to microvilli in the wing disc epithelium.

Upon a closer inspection of the wing imaginal disc epithelium at the ultrastructural level using transmission electron microscopy (TEM), we noticed that posterior cells of $h h>$ PromL $R N A i$ wing discs displayed abnormal microvilli or severely atrophic protrusions (with a length 98\% shorter than that of microvilli from wild-type (wt) discs) (Fig. 1c,d; Supplementary Fig. 3a-c). In contrast, in anterior $w t$ cells, microvilli length, density and morphology was similar to that of $w t$ discs (Fig. 1c,d; Supplementary Fig. 4 a,b). These findings point to the requirement of PromL for the maintenance of the proper microvilli architecture and integrity in the wing disc epithelium, and are consistent with recent observation that the homologous Prominin-1 modulates microvilli architecture in mammalian cells ${ }^{19}$. Furthermore, our results establish that members of the Prominin protein family are key determinants of microvilli formation and integrity, putting forward the existence of a conserved function of the Prominin protein family trough evolution.

Having established that PromL localises to and is required for the maintenance and integrity of microvilli, we explored its relationship with Hh localization and its signaling properties. We first examined Hh distribution in $w t$ and in posterior ( $h h>)$ or dorsal (ap >) 
PromL depleted compartments of wing imaginal discs, and found that in $w t$ discs, Hh distributed at the uppermost apical surface above E-cad (Fig. 1e). No such distribution was observed in PromL mutant compartment in which microvilli were absent or misshapen (Fig. 1c), and lacking therefore a structural support for the apical distribution of Hh. Consistently, in PromL depleted cells, Hh was detected at the basolateral at the level of Dlg, and subapical to

104 E-cad (Fig. 1f; Supplementary Fig. 2b). A subsequent quantitative analysis of the signal distribution revealed a substantial reduction of Hh staining at microvilli in posterior cells (Fig. 1g,h; methods), further supporting that the apical distribution of Hh relies on the presence of intact microvilli.

To examine the consequences of the subcellular redistribution of Hh in PromL depleted cells interferes on Hh signaling properties, we next monitored the expression of long- and shortrange Hh target genes simultaneously in $h h>$ PromL $R N A i$ discs. In wing imaginal discs, $d p p$ lac $\mathrm{Z}$ reporter expression reflects the apical secretion and the long-range Hh signaling, whereas

112 En and Ptc expression is attributed to Hh short-range activity mediated by its basolateral 113 secretion $^{3-5}$. We found that in PromL KD discs Hh long-range signaling is impaired, as 114 evidenced by a severe decrease of $d p p$-lacZ reporter expression ranging from $60-65 \%$. In 115 contrast the expression of the short-range target genes, En and Ptc, remained unaltered (Fig. 1i, 116 j; Table 1; Supplementary Fig. 2d). These results consistently establish that PromL and hence 117 intact microvilli, are not only required for the apical distribution of Hh but also critically 118 involved in Hh long-range signaling in the wing disc epithelium.

To further validate this hypothesis, and substantiate the requirement of microvilli integrity for Hh signaling as suggested by our observations (Fig. 1b-j), we chose to analyze the

121 effect of Dispatched (Disp), a positive regulator of Hh secretion and trafficking ${ }^{5,6,20}$, on the

122 microvillar localisation of Hh. We reasoned that in disp mutant discs, in which Hh is retained 123 in producing cells, and $d p p-l a c Z$ expression is severely restricted (Fig. 2a; Table 1), the 
124 distribution of Hh to microvilli might also be impaired. An analysis of Hh signal showed that

125 in disp mutant discs Hh consistently distributed to the apical, at the level of E-cad, but not Hh

126 to microvilli (Fig. 2b,d), at odds with $w t$ discs, in which Hh distributed to microvilli and

127 colocalised with PromL (50\% of the uppermost apical Hh signal) (Fig. 2c,d). Accordingly,

128 long-range Hh signaling is impaired in disp mutants (Fig. 2a). We also noticed that PromL

129 signal, in anterior and posterior compartment, was more punctate and irregularly spiky, pointing

130 to a shortening of microvilli (Fig. 2b). Additional ultrastructural analysis of disp mutant discs

131 indeed showed shorter and far less microvilli (66\% of that of wt), with an average of $2.9 \pm 1.16$

132 microvilli per cell (Fig. 2d; Supplementary Fig. 3c). Together these experiments indicate that

133 in addition to its role on Hh secretion and trafficking, Disp modulates microvilli architecture,

134 reinforcing again the idea that a proper organization of apical membrane and the maintenance

135 of microvilli architecture is critical for Hh-long-range signaling.

The maintenance of the apical plasma membrane structure relies on the asymmetrical

137 distribution of specific phospholipids between the outer and inner leaflets of plasma

138 membranes ${ }^{21}$. This is controlled by ATP8B, a putative aminophospholipid translocase, whose

139 depletion perturbs apical membrane organization, stereocilia and microvilli integrity ${ }^{22,23}$. It is

140 thus expected that abrogation of ATP8 activity can perturb microvilli organization and hinder

141 Hh long-range signaling also in the wing imaginal disc. Consistent with this hypothesis, the

142 qualitative and quantitative TEM analysis revealed that ATP8B depletion in posterior cells

143 ( $h$ h $>A T P B 8 R N A i)$, resulted in an alteration of microvilli number, length, and morphology, and

144 perturbed the characteristic dot-like staining pattern of PromL indicative of the proper

145 microvilli integrity (Fig. 3a-c; Supplementary Fig. 1e). This was accompanied by a subapical

146 redistribution of $\mathrm{Hh}$, an impediment of PromL/Hh colocalisation, and a reduction of $d p p$

147 expression in recipient cells as a consequence of impaired long-range Hh signaling, but no effet

148 of En and Ptc expression was observed (Fig. 3c-e; Table 1). However, ATP8B depleted cells 
149 show well developed adherent junctions, confirming their ability to polarize (Fig. 3a). Hence,

150 loss of ATP8B activity may affect the proper distribution of PromL to microvilli, by altering

151 the phospholipid composition of the plasma membrane, or by potentially decreasing the levels

152 of the plasma membrane phospholipid phosphatidylinositol 4,5-bisphosphate ( $\left.\mathrm{PIP}_{2}\right)^{24}$.

153 As $\mathrm{PIP}_{2}$, is enriched at the apical plasma membrane of epithelial cells, regulates the

154 interaction of signaling proteins with actin-binding proteins, and promotes the bundling

155 function of villin, it could thereby modulate the formation of actin filaments structures and

156 cellular protrusions, including microvilli (Kumar). To test this possibility, we silenced two

157 actin-binding proteins, Quail (Qua) (ap>Qua RNAi), the Drosophila villin-like protein ${ }^{25}$, or

158 Fimbrin (Fim) ( $h$ h>Fim RNAi) in Drosophila imaginal discs. In both conditions, PromL levels

159 were drastically reduced (Fig. 3 f,g), and microvilli, when present, were strongly defective (Fig.

1603 b,d,h,i; Supplementary Fig. 4c). As expected, Hh long-range signaling was impaired to similar extents in both genetic backgrounds, as confirmed by the subapical distribution of $\mathrm{Hh}$ and by

162 the reduced $d p p$ expression in target cells (Fig 3f,g,j; Supplementary Fig. 3; Table 1). This 163 indicates that the disassembly of actin filaments caused profound structural alterations on the

164 microvillar architecture and put forward the positive contribution of actin cross-linking factors

165 in this process. Moreover, this reveals that an active interplay between PromL and cytoskeleton components contributes to the biogenesis and maintenance of microvilli that are critical for $\mathrm{Hh}$ -

167 long-range signaling.

How do microvilli contribute to Hh long-range signaling? While previous work has

169 established that microvilli give rise to Prominin-containing EVs ${ }^{10,11}$, their contribution to 170 intercellular communication during development was never directly demonstrated. Considering

171 the requirement of microvilli for Hh long-range signaling (as shown above), we reasoned that

172 EVs could bud from microvilli and serve as a means to transport Hh to distant recipient cells.

173 To test this assumption, we performed an EM analysis of serial sectioning of $w t$ wing imaginal 
174 discs epithelial cells. We noticed the existence of small vesicular structures of 60 to $150 \mathrm{~nm}$

175 diameter, often clustered at the vicinity of microvilli, but also free within the lumen (Figure 4

176 a,b; Supplementary 4d; methods). We also observed that MVB with intraluminal vesicles (ILV)

177 of smaller diameter $(25-40 \mathrm{~nm})$, were consistently found at close vicinity of the basolateral

178 plasma membranes (Fig. 4a, lower right panel; Supplementary 4e), but not at the apical site,

179 indicating that MVBs may not be prone to an apical secretion of "endosome-derived"

180 exosomes $^{2}$. Importantly, such vesicular structures were not detected in discs depleted for

181 PromL, ATP8B, Qua, Fim, or disp mutant discs, in which microvilli are absent or atrophic, and

182 dpp-lacZ expression is substantially decreased (Fig. 1c, 2e, 3a,h,i; Table 1), underscoring the

183 correlation between the presence of microvilli, the occurrence of these secreted vesicles and $\mathrm{Hh}$

184 long-range activity. A closer inspection of the microvilli in $w t$ discs, revealed the presence of

185 buds at the microvillar membrane (Fig. 4c). Electron tomography and 3D-reconstructions of

186 the apical plasma membrane confirmed the existence of buds still connected to the microvillar

187 membrane, but also isolated free vesicles within the extracellular space certainly after fission

188 (Fig. 4d, Supplementary Movies 1 and 2). Together, these results substantiate microvilli as a

189 site of EV biogenesis and demonstrate that the active interplay between PromL, actin cross-

190 linking proteins, and microvilli lipids is critical for the maintenance of microvilli integrity and

191 the biogenesis of EVs. Consistent with these findings, Prominin-1 containing EVs were shown

192 to originate from the microvilli membrane and cilia of neuroepithelial and neural progenitors

193 cells respectively ${ }^{10,26}$. In agreement with our findings, actin mediate EV release from the tip of

194 cilia $^{27}$, and dATP8B protein is concentrated in the cilia of olfactory neuron dendrites ${ }^{28}$ where

195 it could play similar roles as the one shown here in microvilli.

196 Given that PromL is critical for microvilli biogenesis, and that $\mathrm{Hh}$ distributed to

197 microvilli where it colocalises with PromL (Fig. 1g,h; 2c,d; 3d), we reasoned that an increase

198 of Hh long-range activity might be correlated with an increase of EV biogenesis. To test this 
assumption, we examined the consequences of the overexpression of PromL-GFP in Hh producing cells $(h h>$ PromL-GFP). EM analysis revealed a substantially increased of EV number (35\% as compare to $w t$ ) in PromL-GFP discs, which resulted in a slight but not significant reduction in microvilli number and length (Fig. 4e,f; Supplementary Fig. 4b). We also found that overexpression of PromL-GFP promoted a massive Hh release into the lumen, which correlated with a significant expansion of the $d p p$ expression domain (Fig. 4g,h; Table PromL localisation to microvilli, EV biogenesis and Hh long-range activity. and PromL protein are released into the extracellular medium. To validate this hypothesis, we investigated their occurrence and their potential properties in wing discs overexpressing 210 PromL-GFP and Hh tagged with red fluorescent protein (Hh-RFP) in Hh producing cells (hh>Hh RFP; PromL GFP). In a first round of experiments, we set out to investigate the distribution of Hh-GFP and Prom-GFP using immunoelectron microscopy (IEM), for a qualitative analysis. In agreement with our above observations (Fig. 1g,h; 2c,d; 3d,) both 214 proteins localised not only to microvilli (Fig. 5a), but also on EVs (Fig. 5 b). To elucidate their 215 dynamics and their functional roles, we investigated their properties in vivo. Live imaging of hh>Hh RFP; PromL GFP discs revealed that the majority (80\%) of EVs released by the

217 posterior compartment was positive for Hh-RFP and Prom-GFP, whereas the others only 218 displayed Hh-RFP (Fig. 5c-e). These EVs were not static but engaged in a dynamic movement 219 and trafficked associated to one another within the extracellular space (Fig. 5f,g; supplementary 220 Fig. 5a; Supplementary Movies 3). These EVs are very likely the extracellular carriers of Hh, 221 facilitating thereby its long-range activity. These results are consistent with our hypothesis that 222 microvilli of the wing disc epithelium are the site of generation of small EVs, that are critically 223 involved in the transport of Hedgehog across to facilitate its long-range activity. 
Understanding the origin and function of EVs in vivo during development has so far been limited especially because of a shortage of methods suitable to demonstrate causality in vivo ${ }^{10,11}$. Taking advantage of complementary electron and fluorescence microscopy, live interaction with lipids and cytoskeleton components, is a key determinant of microvilli

230 formation in vivo, whose integrity is critical for the biogenesis of EVs and their signaling role

231 (Fig. 1; Fig. 3). Finally, we uncover that microvilli are the preferential site for the generation 232 of small EVs conveying Hh, - different form exosomes- , revealing the existence of a new 233 potential mechanism mediating Hh long-range signaling in the Drosophila wing imaginal disc 234 epithelium, and unveil their physiological significance in tissue development in vivo. invertebrates and vertebrates, and that the dysregulation of the Hh pathway promotes developmental defects and contributes to several cancer types, it will be of high interest to investigate the contribution of the EV-mediated Hh signaling described in this study to such physiological and pathological processes.

\section{References}

243 1. Briscoe, J. \& Thérond, P. P. The mechanisms of Hedgehog signalling and its roles in

244 development and disease. Nat. Rev. Mol. Cell Biol. 14, 416-429 (2013).

245 2. van Niel, G., D’Angelo, G. \& Raposo, G. Shedding light on the cell biology of 246 extracellular vesicles. Nat. Rev. Mol. Cell Biol. 19, 213-228 (2018).

247 3. Ayers, K. L., Gallet, A., Staccini-Lavenant, L. \& Thérond, P. P. The long-range 248 activity of Hedgehog is regulated in the apical extracellular space by the glypican Dally and 
the hydrolase Notum. Dev. Cell 18, 605-620 (2010).

4. Matusek, T. et al. The ESCRT machinery regulates the secretion and long-range activity of Hedgehog. Nature 516, 99-103 (2014). through dispatched regulates long-range signaling. Dev. Cell 32, 290-303 (2015).

6. Callejo, A. et al. Dispatched mediates Hedgehog basolateral release to form the longrange morphogenetic gradient in the Drosophila wing disk epithelium. Proc. Natl. Acad. Sci. U. S. A. 108, 12591-12598 (2011).

7. Gradilla, A.-C. et al. Exosomes as Hedgehog carriers in cytoneme-mediated transport and secretion. Nat. Commun. 5, 5649 (2014).

259 8. Tanaka, Y., Okada, Y. \& Hirokawa, N. FGF-induced vesicular release of Sonic 260 hedgehog and retinoic acid in leftward nodal flow is critical for left-right determination. Nature 435, 172-177 (2005).

$2629 . \quad$ Vyas, N. et al. Vertebrate Hedgehog is secreted on two types of extracellular vesicles 263 with different signaling properties. Sci. Rep. 4, 7357 (2014).

264 10. Marzesco, A.-M. et al. Release of extracellular membrane particles carrying the stem 265 cell marker prominin-1 (CD133) from neural progenitors and other epithelial cells. J. Cell Sci. 266 118, 2849-2858 (2005).

267 11. Marzesco, A.-M. et al. Release of extracellular membrane vesicles from microvilli of 268 epithelial cells is enhanced by depleting membrane cholesterol. FEBS Lett. 583, 897-902 269 (2009).

270 12. Corbeil, D., Röper, K., Fargeas, C. A., Joester, A. \& Huttner, W. B. Prominin: a story 271 of cholesterol, plasma membrane protrusions and human pathology. Traffic Cph. Den. 2, 82$27291(2001)$.

273 13. Fargeas, C. A., Florek, M., Huttner, W. B. \& Corbeil, D. Characterization of 
274 prominin-2, a new member of the prominin family of pentaspan membrane glycoproteins. $J$.

275

276

277

278

279

280

281

282

283

284

285

286

287

288

289

290

291

292

293

294

295

296

297

298

Biol. Chem. 278, 8586-8596 (2003).

14. Zelhof, A. C., Hardy, R. W., Becker, A. \& Zuker, C. S. Transforming the architecture of compound eyes. Nature 443, 696-699 (2006).

15. Demontis, F. \& Dahmann, C. Apical and lateral cell protrusions interconnect epithelial cells in live Drosophila wing imaginal discs. Dev. Dyn. Off. Publ. Am. Assoc. Anat. 236, 3408-3418 (2007).

16. Morin, X., Daneman, R., Zavortink, M. \& Chia, W. A protein trap strategy to detect GFP-tagged proteins expressed from their endogenous loci in Drosophila. Proc. Natl. Acad. Sci. U. S. A. 98, 15050-15055 (2001).

17. D'Alterio, C. et al. Drosophila melanogaster Cad99C, the orthologue of human Usher cadherin PCDH15, regulates the length of microvilli. J. Cell Biol. 171, 549-558 (2005).

18. Schlichting, K., Wilsch-Bräuninger, M., Demontis, F. \& Dahmann, C. Cadherin

Cad99C is required for normal microvilli morphology in Drosophila follicle cells. J. Cell Sci. 119, 1184-1195 (2006).

19. Thamm, K. et al. Prominin-1 (CD133) modulates the architecture and dynamics of microvilli. Traffic Cph. Den. 20, 39-60 (2019).

20. Burke, R. et al. Dispatched, a novel sterol-sensing domain protein dedicated to the release of cholesterol-modified hedgehog from signaling cells. Cell 99, 803-815 (1999).

21. Simons, K. \& Ikonen, E. Functional rafts in cell membranes. Nature 387, 569-572 (1997).

22. Stapelbroek, J. M. et al. ATP8B1 is essential for maintaining normal hearing. Proc. Natl. Acad. Sci. U. S. A. 106, 9709-9714 (2009).

23. Verhulst, P. M. et al. A flippase-independent function of ATP8B1, the protein affected in familial intrahepatic cholestasis type 1, is required for apical protein expression and 
microvillus formation in polarized epithelial cells. Hepatol. Baltim. Md 51, 2049-2060

300

301

302

303

304

305

306

307

308

309

310

311

312

313 43-71 (2017). (2010).

24. Liu, Y.-C. et al. The Drosophila melanogaster phospholipid flippase dATP8B is required for odorant receptor function. PLoS Genet. 10, e1004209 (2014).

25. Mahajan-Miklos, S. \& Cooley, L. The villin-like protein encoded by the Drosophila quail gene is required for actin bundle assembly during oogenesis. Cell 78, 291-301 (1994). 26. Dubreuil, V., Marzesco, A.-M., Corbeil, D., Huttner, W. B. \& Wilsch-Bräuninger, M. Midbody and primary cilium of neural progenitors release extracellular membrane particles enriched in the stem cell marker prominin-1. J. Cell Biol. 176, 483-495 (2007).

27. Nager, A. R. et al. An Actin Network Dispatches Ciliary GPCRs into Extracellular Vesicles to Modulate Signaling. Cell 168, 252-263.e14 (2017).

28. Ha, T. S., Xia, R., Zhang, H., Jin, X. \& Smith, D. P. Lipid flippase modulates olfactory receptor expression and odorant sensitivity in Drosophila. Proc. Natl. Acad. Sci. U. S. A. 111, 7831-7836 (2014).

29. Strutt, H. et al. Mutations in the sterol-sensing domain of Patched suggest a role for vesicular trafficking in Smoothened regulation. Curr. Biol. CB 11, 608-613 (2001).

30. Gallet, A., Rodriguez, R., Ruel, L. \& Therond, P. P. Cholesterol modification of hedgehog is required for trafficking and movement, revealing an asymmetric cellular response to hedgehog. Dev. Cell 4, 191-204 (2003).

31. Mastronarde, D. N. Dual-axis tomography: an approach with alignment methods that preserve resolution. J. Struct. Biol. 120, 343-352 (1997).

32. Hurbain, I., Romao, M., Bergam, P., Heiligenstein, X. \& Raposo, G. Analyzing Lysosome-Related Organelles by Electron Microscopy. Methods Mol. Biol. Clifton NJ 1594, 


\section{Acknowledgments}

We thank A. Plessis and Ph. Vernier for insightful discussions. C. Dahmann, X., D. Godt, the

Genetics Resource Center, and Bloomington stock centers for reagents. Vincent Fraisier from

328 the Imaging platform UMR144; The Cell and Tissue Imaging (PICT-IBISA), Institut Curie,

329 member of the French National Research Infrastructure France-BioImaging (ANR10-INBS-

330 04). This work was supported by the French Government (National Research Agency, ANR)

331 through the Investments for the Future LABEX SIGNALIFE (ANR-11-LABX-0028-01),

332 LabEx CelTisPhyBio (ANR-11-LABX-0038), ANR (ANR-15-CE13-0002-01), Ligue Contre

333 le Cancer ' Equipe labellisée 2016' to P.P.T, and by the Fondation pour la Recherche Médicale:

334 DEQ201110421324.

335

\section{Authors contributions}

337 Conceptualization: P.P.T, G.R, and G.D.; Methodology: I.H, M.R, L.S., L.R., and G.D.;

338 Software: A.S.M.; Formal analysis: I.H, A.S.M, and G.D.; Investigation: I.H., M.R., L.R., G.D.;

339 Resources: R.B., P.P.T., G.R.; Writing - Original Draft: G.D.; Writing - Review \& Editing:

340 I.H., R.B., P.P.T., G.R. and G.D.; Supervision: G.D.; Funding acquisition G.R. and P.P.T.

\section{Competing interests}

343 The authors declare no competing interests.

\section{Data and materials availability}

346 all data is available in the manuscript or the supplementary information.

\section{$348 \quad$ Figure legends}


Figure 1. Hh long-range activity relies on microvilli integrity. a, Schematic of the larval wing imaginal disc epithelium and a transverse section. The disc is oriented anterior (A) to the left, posterior $(\mathrm{P})$ to the right. The wing imaginal disc consists of two layers of cells with apical surfaces facing each other: the columnar epithelium (CE) of the wing disc pouch overlayered by the squamous epithelium (SE), separated by the extracellular space (lumen). Microvilli cover the apical surface of the wing imaginal disc. $\mathbf{b}, \mathbf{e}-\mathbf{g}, \mathbf{i}, \mathbf{j}$ Single confocal transverse and XY sections of discs of the indicated genotypes stained for PromL (red; white), aPKC (green), Ecad (blue), Hh (green), En (blue; white), Ptc (red; white) and $\beta$-gal (reflecting the expression of the reporter gene dpp-lacZ; green; white). b, Yellow arrows mark the absence of PromL in 358 D compartment. Yellow broken line delineates the apical surface. c, Transmission electron micrograph of $h h>$ PromL RNAi wing disc showing the absence of microvilli PromL depleted for the indicated genotypes (see Supplementary Fig. 4a). Mean \pm SEM analysed by unpaired $t$

362 test, $* * * * p<0.0001$. e, f, Yellow arrows and arrowhead mark apical and subapical Hh 363 distribution in $w t$ and $h h>$ PromL $R N A i$ wing discs respectively. g, Distribution of Hh, and 364 PromL in wt discs. h, Quantification of PromL and Hh colocalisation within the delimited region (see Supplementary information). Mean \pm SEM analysed by unpaired $t$ test $(* * * * p<$ 0.0001) ( $\mathrm{n}=24$ uppermost apical regions, 8 discs). i,j, Confocal XY single section showing Hh 367 target gene expression. Red dots in the insets depict the number of cells expressing En, Ptc and $368 d p p$-lacZ for the shown discs, but representative of all examined discs $(w t \mathrm{n}=8$; PromL RNAi $\mathrm{n}=6$ ) (see Table 1). Broken lines delimit V/D and A/P compartments. Scale bars: $1 \mu \mathrm{m}(\mathbf{c}) ; 20$ $370 \mu \mathrm{m}(\mathbf{b}, \mathbf{e}-\mathbf{h})$.

372 Figure 2. In disp mutant discs microvilli integrity is perturbed. a-c, Single confocal 373 transverse and XY sections of discs of the indicated genotypes stained for Hh (green; white), 
$374 \beta$-gal (red; white), PromL (red; white), and E-cad (blue). a, Hh is retained in P compartment 375 and $d p p$-lacZ expression is reduced to 4 cells (red dots in the inset and Table 1). b, Hh distributes 376 at the apical at the level of E-cad (white arrow). Yellow arrows point to PromL staining 377 reflecting changes in microvillus length, and compare to PromL staining in A $w t$ compartment 378 typical of the intact microvilli organization (c). Yellow arrows point to the similar PromL signal in $\mathrm{A}$ and $\mathrm{P}$ compartments. c, In wt discs, Hh distributed to microvilli. Bottom: shows the colocalisation of both proteins (see Supplementary information). d, Quantification of PromL/Hh colocalisation (see Supplementary information). Mean \pm SEM analysed by unpaired $t$ test $(* * * * p<0.0001)(\mathrm{n}=18 ; 6$ discs $)$. e, Transmission electron micrograph showing shorter 383 microvilli (black arrows) as compare to wt (Fig. 4a). Asterisks depict adherent junctions. f, 384 Graph reporting microvilli length. Mean \pm SEM analysed by unpaired $t$ test $(* * * * p<.0 .0001)$. Scale bar: $20 \mu \mathrm{m} ; 1 \mu \mathrm{m}(\mathbf{e})$. White dashed line delimits A/P compartments.

Figure 3. Lipids and cytoskeleton components contribute to microvilli architecture. a,h,i,

388 Transmission electron micrograph of transverse sections of wing imaginal discs of the indicated genotype showing microvilli in A (black arrows), and adherent junctions (asterisk). Note that in P compartment, microvilli are reduced in size, or absent. b, Histogram of microvilli length. Mean \pm SEM analysed by unpaired t test $(* * * * p<.0 .0001)$. n.s., not significant, $p=0.5230$. $392 \mathbf{c}, \mathbf{e - g}, \mathbf{j}$, Transverse $(\mathbf{c}, \mathbf{f}, \mathbf{g})$ and XY $(\mathbf{e}, \mathbf{j})$ confocal sections of wing imaginal discs from the 393 indicated genotypes stained for PromL (red; white), Hh (green; white), En (blue, white), Ptc 394 (red; white), $\beta$-gal (green, white), wg (blue). c,f,g, Distribution of Hh and PromL for the 395 indicated genotypes. c, Note absence of colocalisation of PromL and Hh in ATP8B depleted 396 cells (white arrow). f,g, White and yellow arrows depict the absence of PromL staining and the 397 basolateral localisation of Hh in D PromL depleted cells respectively. d, Quantification of 398 PromL/Hh colocalisation in discs of the indicated genotypes. Mean \pm SEM analysed by 
unpaired $t$ test $(* * * * p<0.0001)$. Between 8-15 discs were analysed. e,j, Hh target gene expression in discs of the indicated genotypes. Red dots in the insets indicate the cells expressing En, Ptc and $d p p$-lacZ for the shown discs but representative of all examined discs $($ ATP8B RNAi $\mathrm{n}=5$; Quail RNAi $\mathrm{n}=7$; Fim RNAi $\mathrm{n}=6)$ (see Table 1). Black $(\mathbf{a}, \mathbf{h}, \mathbf{i})$ and white broken lines (c,e-g,j) delimit the A/P or V/D compartments. Scale bar: $1 \mu \mathrm{m}(\mathrm{A}, \mathrm{B}) ; 10 \mu \mathrm{m}(\mathrm{C}-$ $\mathrm{L}) ; 20 \mu \mathrm{m}(\mathrm{M})$.

405

Figure 4. Microvilli-derived EVs control Hh long-range signaling. (a,c,e) Transmission electron micrograph images of a transverse section of wing imaginal discs. a, Microvilli (black arrows), adherent junction (red arrowhead). Top right: magnification of the white boxed region showing vesicular structures at the vicinity of the microvilli (asterisks). Bottom right shows an MVB. Yellow arrows point to ILVs. b, Distribution of EVs based on their diameter size $(\mathrm{n}=$ 32). c, EVs are close to microvilli (asterisks), where a bud is also visible (yellow arrow). Note that EVs are less electron dense than sections of microvilli (black arrowheads). d, 3Dreconstruction and modelisation of vesicles budding at microvilli. The buds (red) are still

414 connected to the microvillar membrane (yellow) and free vesicles (red) are detected into the 415 lumen. Right, vesicles 1-3 budding at microvilli correspond to the vesicles in (d). e, 416 Transmission electron micrograph of $h h>$ PromL-GFP disc showing EVs (yellow asterisks) into 417 the lumen and close to microvilli (yellow asterisks). f, Histogram reporting the length of 418 microvilli. Mean \pm SEM analysed by unpaired $t$ test (n.s., not significant), $p=0.4474$. (g,h) $419 a p>$ PromL-GFP (g) or $h$ h $>$ PromL-GFP (h) discs stained for E-cad (blue), Hh (red), GFP 420 (green), and $\beta$-gal (red, white). (g) Hh (yellow arrow) is detected within the lumen (delimited 421 by a white dotted line) in both D and V compartments. (h) XY confocal section. Left, PromL422 GFP is detected in A cells (yellow arrow). Right, cells expressing dpp-lacZ (red dots) in the 
inset. Six to eight discs from each genotype were analysed, (see Table 1).Broken lines delimit the V/D (g) or A/P (h) compartments. Scale bar: $0.2 \mathrm{~nm}(\mathbf{a}, \mathbf{c}-\mathbf{e}) ; 20 \mu \mathrm{m}(\mathbf{g}, \mathbf{h})$.

Figure 5. Hh-PromL-EVs move into the extracellular field. a, Ultrathin cryosection of hh>Hh RFP; PromL-GFP disc immunolabeled for Hh (PAG 10; black arrows) and GFP (PAG

PromL-GFP disc recorded every 3 min for $90 \mathrm{~min}$. Bottom, magnification of the inset showing examples of Hh/PromL-containing EVs (white circles) or Hh-containing EVs (yellow circles). gradually increased with time and is of $10 \mu \mathrm{m}$ in $80 \mathrm{~min}$ for the shown vesicle. The manual $5 \mu \mathrm{m}(\mathbf{d}, \mathbf{f})$.

Table 1. En, Ptc and ddp-lacZ expression in different genotypes.

Mean number of cells expressing genes

\begin{tabular}{|c|c|c|c|}
\hline Genotype & $\overline{E n}$ & Ptc & dpp-lacZ \\
\hline $\begin{array}{ll}w t \quad(\mathrm{n}=12) \\
\end{array}$ & $2( \pm 0.42)$ & $3.7( \pm 0.4)$ & $7.6( \pm 0.5)$ \\
\hline $\mathrm{hh}>$ PromL RNAi ${ }^{51957 G D}$ & $2^{b}$ & $3.8^{\mathrm{b}}( \pm 0.3)$ & $2.6^{\mathrm{a}}( \pm 0.5)$ \\
\hline $\mathrm{hh}>$ PromL RNAi $102612 \mathrm{KK}$ & $2^{b}( \pm 0.4)$ & $3.7^{\mathrm{b}}( \pm 0.4)$ & $3^{\mathrm{a}}( \pm 0.3)$ \\
\hline disp $p^{S O 37707}$ & n.d. & n.d. & $2.5^{\mathrm{a}}( \pm 0.5)$ \\
\hline hh>ATP8B RNAi ${ }^{102648 K K}$ & $1.92^{\mathrm{b}}( \pm 0.29)$ & $3.7^{\mathrm{b}}( \pm 0.4)$ & $4^{\mathrm{a}}( \pm 0.3)$ \\
\hline
\end{tabular}




\begin{tabular}{|c|c|c|c|}
\hline hh>Qua RNAiGD $51957 G D$ & $1.92^{\mathrm{b}}( \pm 0.3)$ & $4^{b}$ & $3.8^{\mathrm{a}}( \pm 0.3)$ \\
\hline hh>Qua RNAi ${ }^{100856 K K}$ & $1.91^{\mathrm{b}}( \pm 0.3)$ & $3.8^{b}( \pm 0.3)$ & $4^{\mathrm{a}}( \pm 0.3)$ \\
\hline hh $>$ Fim RNAi ${ }^{47514 G D}$ & $1.9^{\mathrm{b}}( \pm 0.3)$ & $3.9^{\mathrm{b}}( \pm 0.4)$ & $4^{\mathrm{a}}( \pm 0.3)$ \\
\hline$h \mathrm{~h}>$ Fim RNAi ${ }^{47511 G D}$ & $1.97^{\mathrm{b}}( \pm 0.3)$ & $3.8( \pm 0.4)$ & $4.2^{\mathrm{a}}( \pm 0.4)$ \\
\hline$h \mathrm{~h}>$ Fim RNAi ${ }^{46028 G D}$ & $2.2^{\mathrm{b}}( \pm 0.5)$ & $3.9^{b}( \pm 0.3)$ & $4.1^{\mathrm{a}}( \pm 0.4)$ \\
\hline $\mathrm{hh}>$ Fim RNAi ${ }^{46029 G D}$ & $1.97^{\mathrm{b}}( \pm 0.3)$ & $3.7^{\mathrm{b}}( \pm 0.4)$ & $4.1^{\mathrm{a}}( \pm 0.4)$ \\
\hline hh>UASPromL GFP & n.d. & $3.9^{\mathrm{b}}( \pm 0.2)$ & $10.3^{\mathrm{a}}( \pm 0.4)$ \\
\hline
\end{tabular}

458

459 The number of cells expressing En, Ptc and dpp-lacZ was assessed in the rectangles in Fig. 1i,j;

460 Fig. 2a; Fig. 3e,j; Fig.4h, Supplementary Fig. 2d; Supplementary Fig. 3a-e) in at least 8 different

461 discs for each genotypes. Mean \pm SEM analyzed by unpaired $t$ test: ${ }^{\mathrm{a}}(* * * * p<0.0001) ;{ }^{\mathrm{b}}$ (n.s., 462 not significant).n.d. not determined. 
Figure 1

a wing pouch

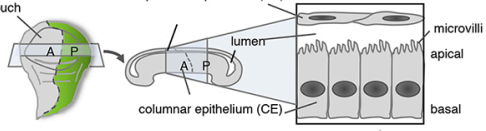

b

ap>PromL RNAi $51957 G 0$

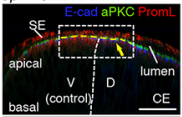

c

hh>PromL RNAi ${ }^{51957 G 0}$

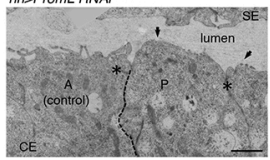

d

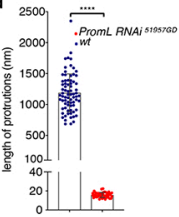

e

wt
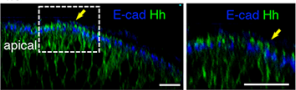

f

$h$ hh PromL RNAi 5195760
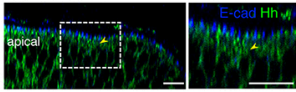

g

$h h>$ PromL RNAi 5195790
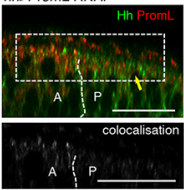

$\mathbf{i}_{\text {hh>PrOmL RNAi 51957GD }}$
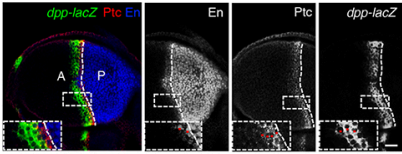

j $w t$
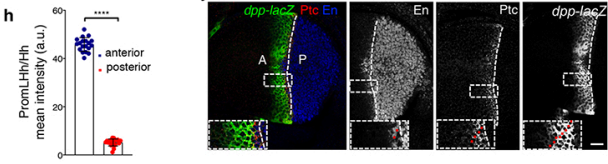
Figure 2

a

disp $p^{5037707}$
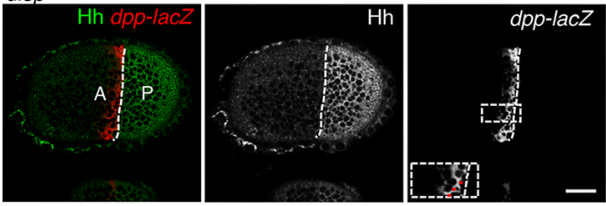

b disp 5037707
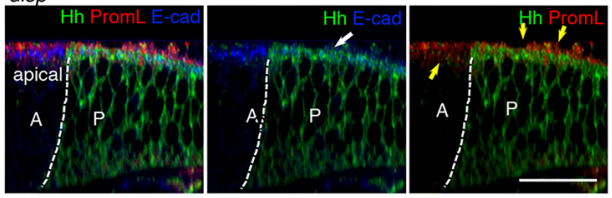

C $w t$
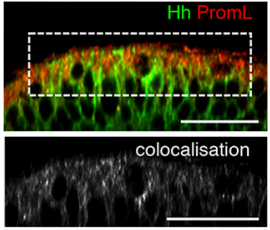

e $\quad \operatorname{disp} p^{5037707}$

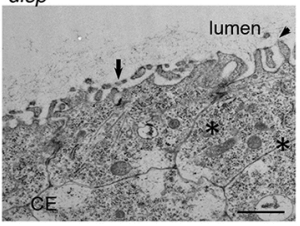

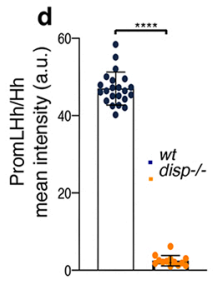

f

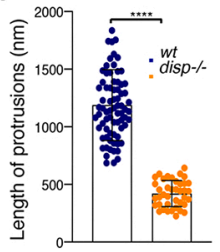


Figure 3

a

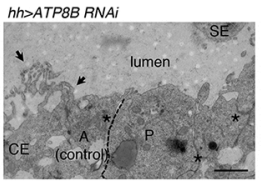

c
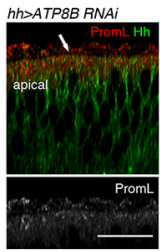

d

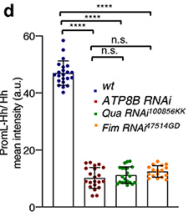

e

f

g $h h>A T P 8 B R N A i$
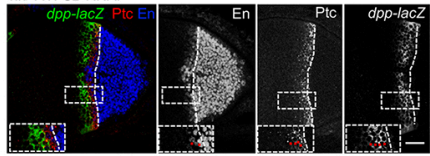

ap>Qua RNA; ${ }^{100856 \kappa \kappa}$
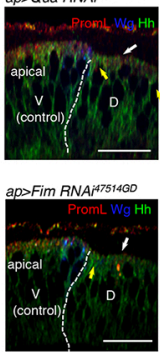

h
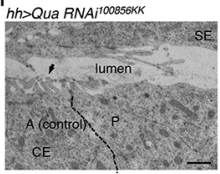

i

$a p>$ Fim $R N A 4751460$

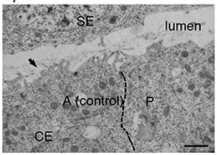

j $h h>Q u a R_{N A i^{100856 K K}}$
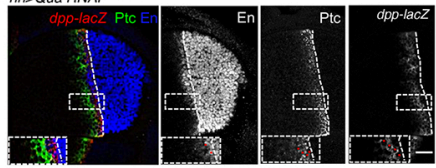
Figure 4

a $w t$

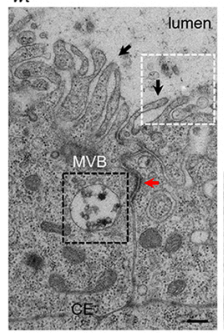

C $w t$

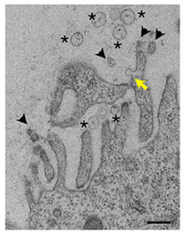

e $h h>$ PromL-GFP

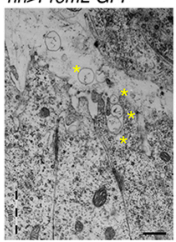

b

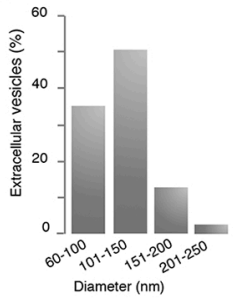

d $w t$

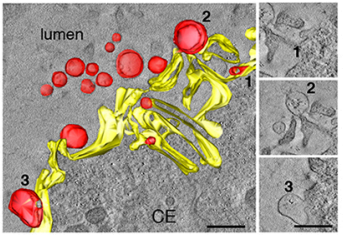

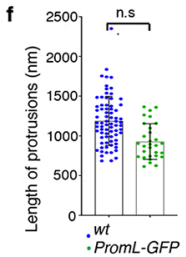

g

ap $>$ PromL-GFP

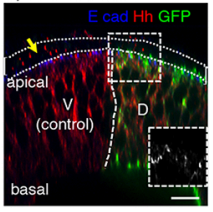

h

$h h>$ PromL-GFP

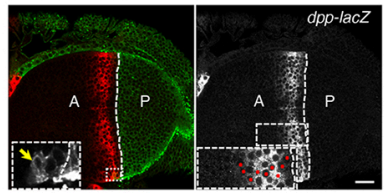


Figure 5

a

$h h>H h-R F P$; PromL-GFP

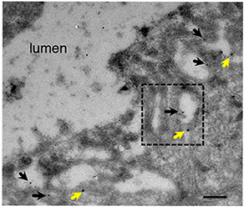

Hh-PAG $10 \mathrm{~nm}$

GFP.PAG $15 \mathrm{~nm}$

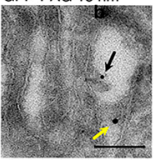

b

hh>Hh-RFP; PromL-GFP

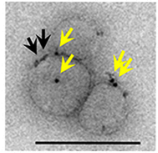

c

$h h>H h$-RFP; PromL-GFP

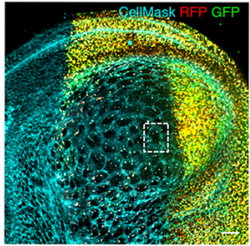

$\mathbf{f}$

$h h>H h-R F P$; PromL-GFP

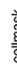

v

뭄
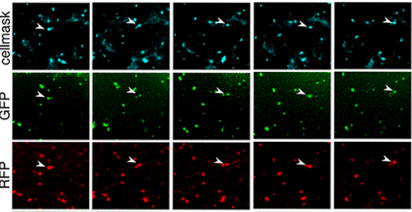

맘

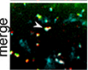

0
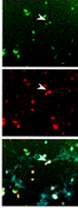

20
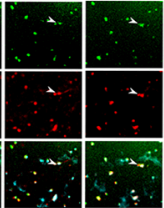

40 time (min)
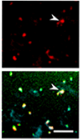

60 d

$h h>H h-R F P$; PromL-GFP
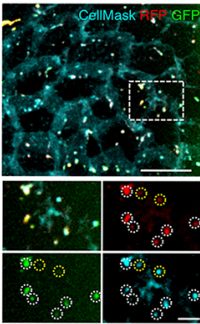

e

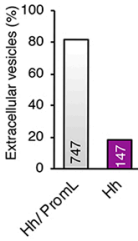

g

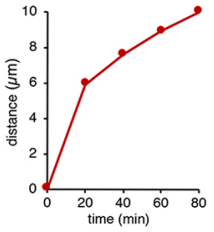


Supplementary Figure 1

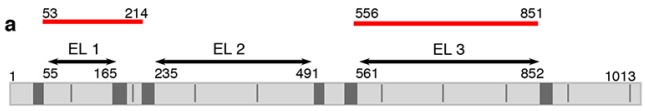

b ap>PromL-GFP

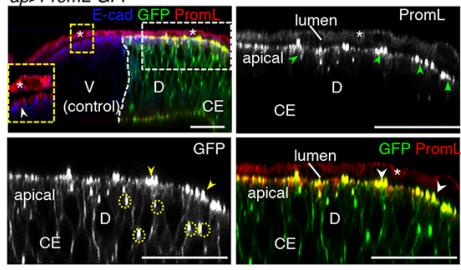

C
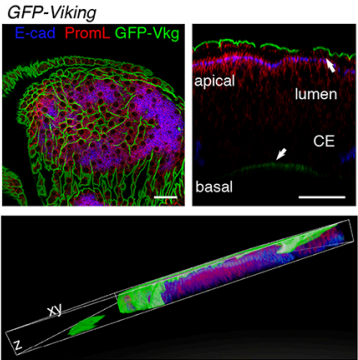

$\mathrm{e}_{w t}$

E-cad PromL DIg

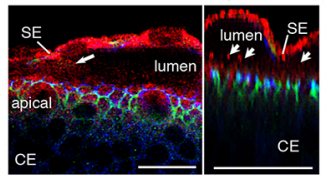

d

ap>PromL-GFP
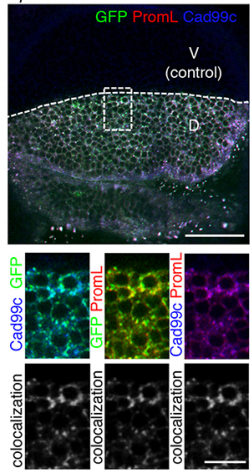


\section{Supplementary Figure 2}

a

$h h>$ PromL RNA ${ }^{51957 a D}$

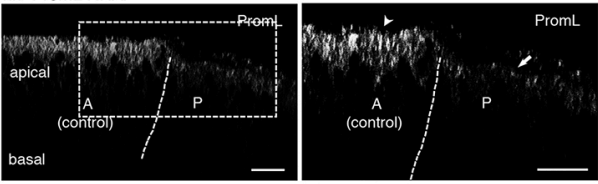

b

ap>PromL RNA $51957 G D$
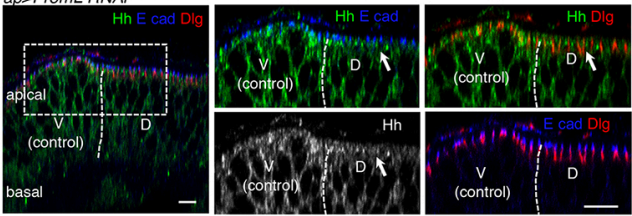

C

$h h>$ PromL RNA 5 1957GD
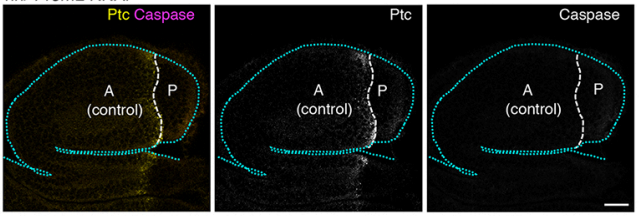

d

$h h>$ PromL RNAi102648KK
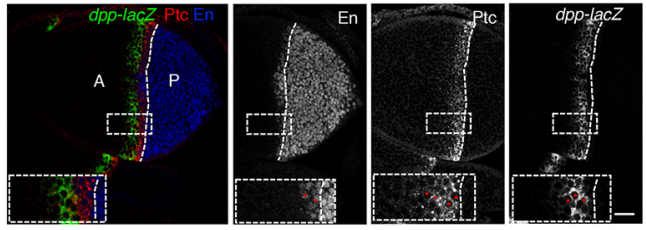
Supplementary Figure 3

a

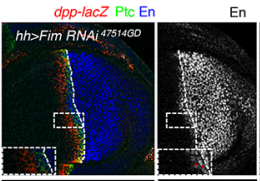

b
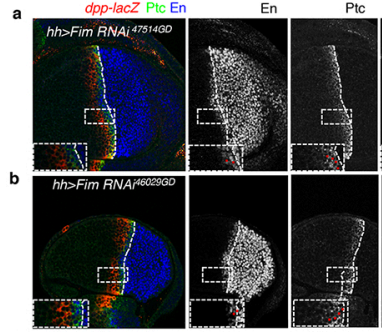

dpp-lacZ

c

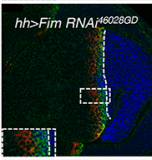

d

$h h>$ Fim RNA $175+7100$

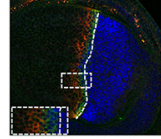

e
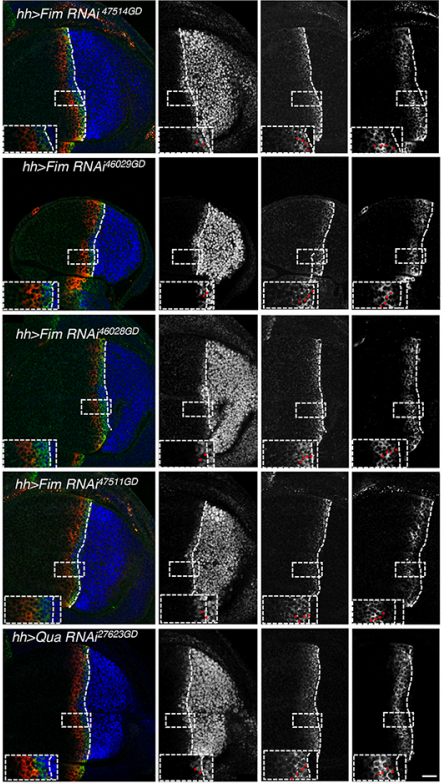
a

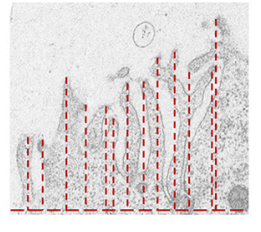

c

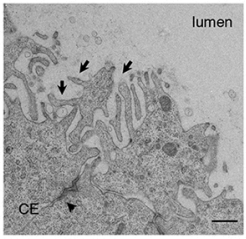

b

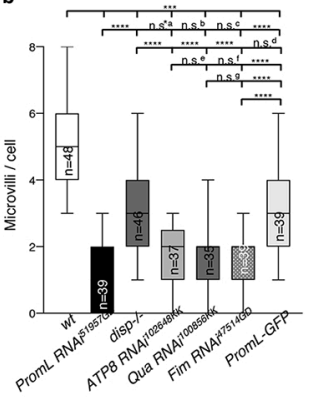

d
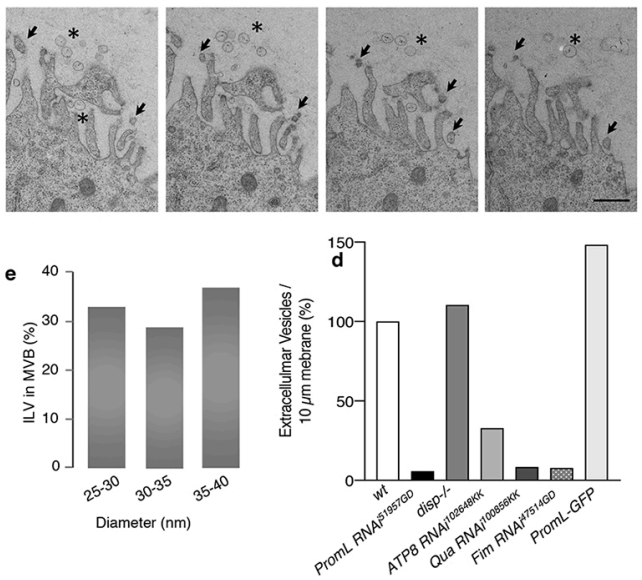
Supplementary Figure 5
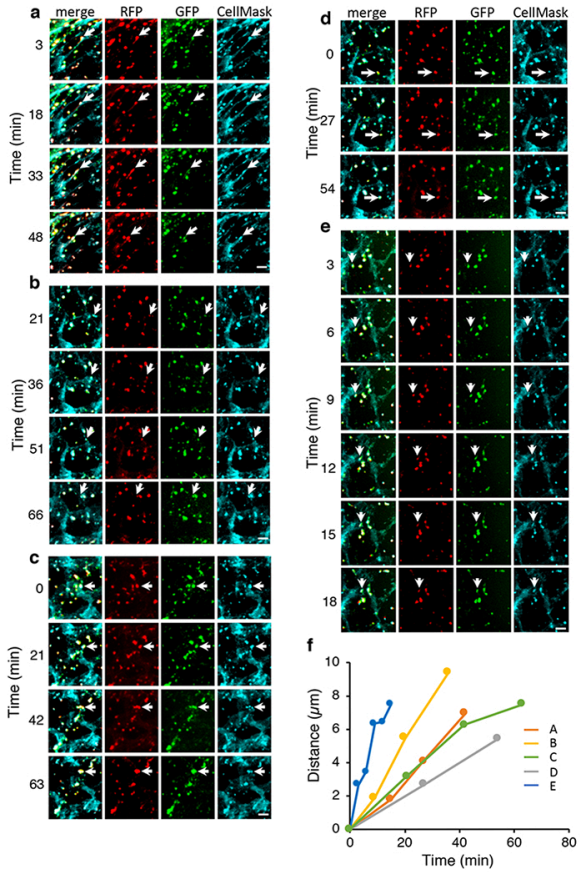